\title{
Evolution in Surgical Treatment of Vestibular Schwannomas
}

\author{
Marcos Tatagiba ${ }^{1} \cdot$ Florian H. Ebner $^{1,2} \cdot$ Taishi Nakamura $^{1,3} \cdot$ Georgios Naros $^{1}$
}

Accepted: 19 July 2021 / Published online: 3 September 2021

(c) The Author(s) 2021

\begin{abstract}
Purpose of Review Management of vestibular schwannomas (VSs) is multimodal and include watchful observation, radiation treatment, and surgery. Over the past decades, a shift in treatment strategy toward radiation treatment has gradually displaced surgery from the main treatment option for VS. In recent years, however, surgery has been further refined by developments of microsurgical and endoscopic techniques and advances in intraoperative application of neuroprotective drugs. This article presents outcomes of modern surgical treatment of VS in the era of radiosurgery and reviews recent published advancements relevant to VS management.

Recent Findings Following VS surgery, excellent tumor resection rates and cranial nerve outcomes were achieved in a consecutive series of 572 adult patients with mean postoperative follow up of 4 years. Innovations in surgical technique include endoscopic technique as additional tool to microsurgery, exploration of semi-sitting position for large tumors, and intraoperative use of vasoactive agents as neuroprotective strategy.

Summary Despite great developments in radiation treatment of VS, surgery remains the key solution for the majority of the cases in order to achieve cure of the disease, long-term tumor control, and preservation of cranial nerve function at long-term.
\end{abstract}

Keywords Vestibular schwannoma $\cdot$ Facial nerve $\cdot$ Surgical technique $\cdot$ Retrosigmoid approach $\cdot$ Outcome $\cdot$ Tumor recurrence

\section{Introduction}

Vestibular schwannomas (VSs) belong to the most common benign tumors of the skull base, accounting for more than $90 \%$ of all non-malignant nerve sheath tumors [1]. While VS has a relatively constant histological appearance with low mitosis rates, radiological presentation may vary considerably (Fig. 1): from large to small, from solid to cystic, from compact with high cellular density to soft and well vascularized, from medial located to invading the petrous bone and filling the entire internal auditory canal (IAC). This variability poses a major challenge on decision-making regarding the best treatment

This article is part of the Topical collection on OTOLOGY: Vestibular Schwannomas

Marcos Tatagiba

marcos.tatagiba@med.uni-tuebingen.de

Florian H. Ebner

florian.ebner@krupp-krankenhaus.de

Taishi Nakamura

n_taishi_yh@hotmail.co.jp

Georgios Naros

georgios.naros@med.uni-tuebingen.de strategy. Management of VS may include watchful observation, radiation treatment, surgery, or a combination of them. Over the past decades, a gradual change in VS management toward radiation has dethroned surgery from its position as treatment of choice. However, no randomized trial comparing radiotherapy and surgery has been conducted so far, and the power of the few clinical retrospective series does not allow to formulate stronger level of evidence higher than class III [2]. Recent studies show that long-term oncofunctional results of radiosurgery are poorer than initially reported in the first series which were characterized by short-term follow-up [3, 4•]. At the same time, surgical treatment has been further refined by the evolution of microsurgical and endoscopic techniques [5] and the advances in intraoperative neuroprotective agents [6•].

Department of Neurosurgery and Neurotechnology, Eberhard Karls University of Tuebingen, Hoppe-Seyler-Str. 3 , Tuebingen 72076, Germany

2 Department of Neurosurgery, Alfried Krupp Hospital, Essen, Germany

3 Department of Neurosurgery, Yokohama City University, Yokohoma, Japan 

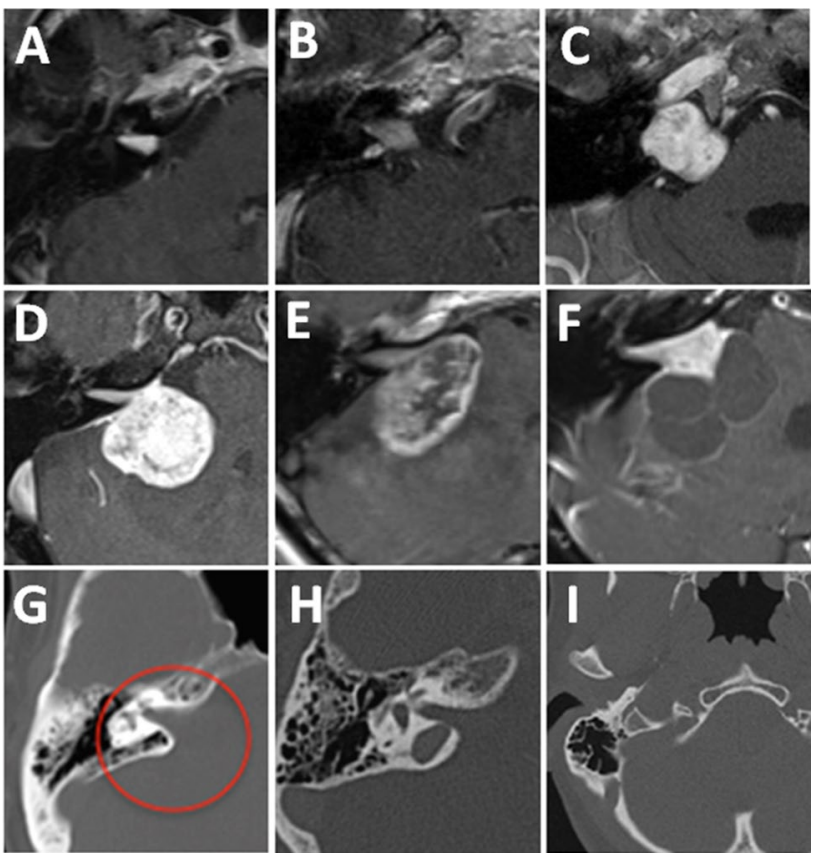

Fig. 1 Imaging in VS. Radiological MR presentation (A-F) of VS may vary considerably: from large to small, from solid to cystic, from compact with high cellular density to soft and well vascularized, from medial located to invading the petrous bone and filling the entire internal auditory canal (IAC). Preoperatively, high-resolution bone window CT is recommended to evaluate the pneumatization of the petrous bone $(\mathbf{G})$ as well as the presence of a high jugular bulb $(\mathbf{H})$ and large emissary veins (I)

This article presents the outcome of contemporary surgical VS treatment in the era of radiosurgery and reviews recent published advancements relevant to VS management. We claim that modern VS surgery remains the key solution in a large number of patients enabling long-term tumor control and function preservation.

\section{Material and Methods}

\section{Patient Cohort}

Since 2004, a total of 2000 VS have been treated at the Department of Neurosurgery and Neurotechnology of the Eberhard Karls University Tuebingen, Germany. In approximately 400 VSs (20\%), radiosurgery or observation strategy has been recommended, and in another 1600 cases $(80 \%)$, the tumor has been removed using the retrosigmoid approach. Major reasons for surgery were as follows: large tumor, growing tumor in young age, tumor with large cystic component, neurofibromatosis 2 (NF2), disabling vestibular symptoms, and patient's individual preference. With approval of the Ethic Committee of the University Hospital Tuebingen, a retrospective study was conducted on 572 consecutive cases of solitary VS operated between 2008 and 2015. Tumors with previous treatments (surgery or radiation), NF2-associated tumors, and pediatric patients were excluded. The following parameters were investigated: tumor resection rate, facial and hearing outcomes, complications, and progression-free survival.

\section{Preoperative Management}

At our institution, there is a standardized preoperative assessment of VS patients. Every VS patient undergoes a MRI of the head (with and without contrast enhancement) and a bone window CT scan of the skull base (Fig. 1). Tumor sizes were classified according to Koos system [7] into T1 to T4. Additionally, a transesophageal echocardiography (TEE) is requested to rule out a patent foramen ovale in patients selected for surgery in semi-sitting position. Pure tone audiography with speech discrimination and auditory evoked potentials are performed to classify hearing according to Gardner and Robertson grading $\left(G \& R^{\circ}\right)$ [8]. Facial nerve function is photo-documented and graded according to the House and Brackmann grading scale $\left({\left.\mathrm{H} \& \mathrm{~B}^{\circ}\right)}^{\circ}[9]\right.$.

\section{Intraoperative Monitoring}

VS surgeries are generally performed under continuous intraoperative neuromonitoring (IONM). IONM encompass the somatosensory- (SSEP) and motor-evoked potentials (MEPs) to the upper and lower extremities, corticobulbar motor-evoked potentials (CoMEPs), and free-running electromyography (EMG) of the affected cranial nerves (CN VII, $\mathrm{XI}, \mathrm{XII}$ ) as well as the brainstem auditory-evoked potentials (BAEPs). To avoid any damage to the cervical spinal cord during the positioning maneuver, particularly for the semisitting position, SSEPS are recorded before and immediately after the patient has been seated and the head fixed with the head clamp. Any significant drop on the SSEP may indicate major spine cord compression that demands positioning adjustments. Major advantage of facial CoMEP in comparison to direct electrical stimulation (DES) is - particularly in large VS - the certainty about facial function even before the facial nerve has been identified and directly stimulated. Any residual hearing $\left(\mathrm{G} \& \mathrm{R}^{\circ} \mathrm{I}-\mathrm{IV}\right)$ demands BAEP recordings to increase the chance of hearing preservation. Deaf patients $\left(\mathrm{G} \& \mathrm{R}^{\circ} \mathrm{V}\right)$ do not receive BAEP recordings. In large VS, additional recording of lower cranial nerves (CN XI, $\mathrm{XII}$ ) is carried out to protect swallowing function.

\section{Microsurgical Technique}

VS surgery is performed either in a supine (SP) or a semisitting position (SSP) depending on tumor size and risk profile of the patient (Fig. 2A). A slightly curved skin incision 


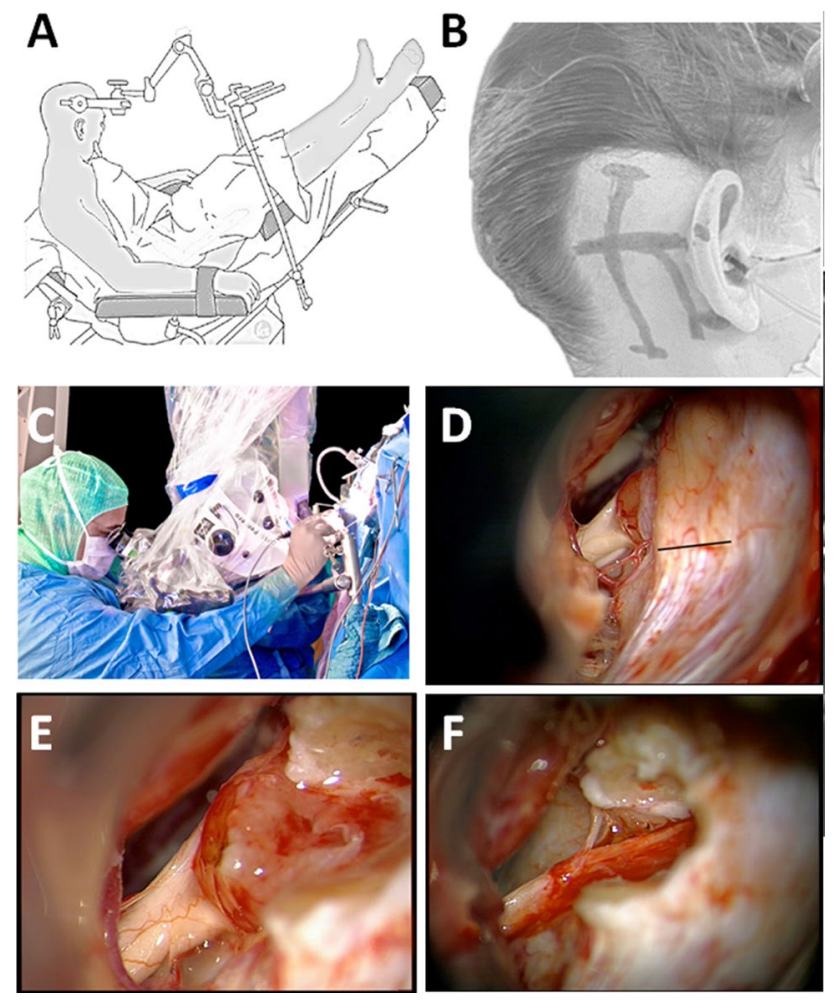

Fig. 2 Surgical techniques. A Schematic presentation of the semisitting position in VS surgery. Please note the position of the legs in relation to the head. Illustrations of the skin incision (B) and the position of the surgeon during semi-sitting surgery (C). After dura opening the Tübingen line (black) is identified (D) representing the position of the internal auditory canal (IAC). Afterward, the IAC is opened with a burr $(\mathbf{E})$ enabling early identification and preservation of the auditory and facial nerves $(\mathbf{F})$

is planned approximately $3-4 \mathrm{~cm}$ behind the ear, above the asterion from the level of the tip of the ear down to the level of the mastoid tip (Fig. 2B). The retrosigmoid approach allows removal of tumors of all sizes; it is compatible with hearing and facial nerve preservation, and it permits in large tumors an excellent control of the lower cranial nerves at the very early beginning of the exposure at the CPA. In young patients, a bone flap is cut and detached along the transversal and sigmoid sinus (Fig. 2C); in older patients, a craniectomy is done after piecemeal bone removal, to avoid dura or sinus wall laceration, since dura adherence is common in elderly patients.

Under the microscope, dura-mater is opened in a straight incision medial and along the sigmoid sinus. No additional dura cuts are needed, which will facilitate later dura closure. The arachnoid layer of basal cisterns is opened and CSF drained. The spatula is introduced from below to above, and gently tilted, so that the inferior space of the CPA appears larger than the superior space. This will prevent rupture of petrosal vein at the early steps of surgery. Cerebrospinal fluid (CSF) is drained in the very first step of the intradural procedure.
After identification of the Tübingen line [10] at the posterior aspect of the petrous bone (Fig. 2D), the IAC is opened with diamond burrs of 3-2 $\mathrm{mm}$. If the patient is deaf and the tumor fills the IAC completely, IAC is opened in its entire length of $10 \mathrm{~mm}$; if the patient still has hearing function, we open approximately $7 \mathrm{~mm}$ of IAC and avoid exposure of the fundus in order to preserve the integrity of the semicircular canals (Fig. 2E). The most lateral part of the IAC is then visualized with $30-45^{\circ}$ endoscopes. Drilling the IAC releases the pressure inside the canal and facilitates the gentle dissection of facial and cochlear nerve (Fig. 2F). Opening of the IAC is always the first step of the intradural procedure, unless the VS is giant and blocks the access to the IAC. The reasons for an early opening of the IAC are as follows: (1) the neurovascular structures in the CPA are still protected by the tumor against bone dust, heat, and irrigation; (2) we gain control of the distal part of the facial and the cochlear nerves; (3) in the final stage of the operation, we can follow the facial nerve at the inferior part of the tumor surface from both sides of the meatus, which facilitates facial preservation while removing the last tumor part. If the thin-slice bone window CT scan shows a high jugular bulb, it can be skeletonized and pushed down to get direct access to the intrameatal tumor portion [11].

At the CPA, tumor is debulked with the ultrasonic surgical aspirator. Before the debulking procedure starts, however, the tumor surface is "mapped" with an electrophysiological probe in order to rule out a variant dorsal position of the facial nerve that may be encountered in $0.6 \%$ of the cases [12]. As soon as tumor mass is significantly reduced, the cleavage plane between tumor surface and surrounding arachnoid is dissected under bimanual work. While the tumor border is held with tumor forceps, the layer containing the arachnoid and the perineurium of the cranial nerves is identified and gently peeled off from tumor with long dissection forceps. Stepwise, inferior, medial, and superior tumor parts are mobilized toward the working corridor and further debulked. In large VS, the lower cranial nerves are early identified and preserved. Above them, along the inferior surface of the tumor the cochlear nerve is identified and carefully dissected and preserved using bimanual dissection with forceps as described above. Medially, facial nerve is identified at its exit zone of the brainstem by direct electrostimulation. In the majority of cases, the facial nerve runs alongside the ventral circumference of the tumor. However, the nerve's track is unpredictable in large tumors and can be displaced in all directions. Therefore, a careful and stepwise dissection from medial to lateral following the nerve fibers is mandatory to preserve facial function.

Because the area of most adhesion for the facial fibers is juxta-meatal, attention must be paid not to cross this point when coming from the brainstem toward the IAC. When this point is reached, further dissection of facial nerve is done in 
contrary direction, which means from the IAC toward the brainstem. The very last piece is elevated and slightly rotated, thus exposing the facial nerve that runs at the inferior-ventral surface of the tumor. This maneuver can be of great help in removing the last tumor remnant under direct control of the facial nerve. The operating field is irrigated several times during tumor dissection with Ringer's solution containing vasoactive substances such as Papaverine.

In case of strong tumor adherence, the last remnant is not removed in order to preserve the integrity of the facial nerve. During the entire surgery within the CPA, use of bipolar coagulation is reduced to a minimum and strictly avoided in proximity to the cranial nerves. Bleedings from arterial branches are selectively coagulated or closed with hemoclips.

Before dura is closed, a $30-45^{\circ}$ endoscope is used to directly depict the fundus of the IAC and rule out any tumor remnant. Endoscopy may also depict opened air cells at the drilled area of IAC that must be sealed off to prevent CSF leak. Opened air cells are plugged off with bone wax under direct endoscopic view to assure complete closure. Then, additional muscle harvested from the neck plus fibrin glue is used to additionally close the IAC. This technique of endoscopeassisted closure of the air cells of IAC with bone wax and muscle + fibrin glue dramatically reduced the incidence of postoperative CSF leakage in our series. Finally, dura is closed in a watertight fashion with a running silk suture. The bone flap or methyl methacrylate is used to close the craniotomy.

\section{Statistical Analyses}

All statistical tests were performed using SPSS (IBM SPSS Statistics for Windows, version 22.0. Armonk, NY: IBM Corp.). Group differences in distribution of clinical attributes are evaluated by Kruskal-Wallis or chi-square tests. The Yates correction is applied when the expected cell frequencies are below 5. Recurrence-free and overall survivals were estimated using the Kaplan-Meier method. Significance was defined as the probability of a two-sided type 1 error being $<5 \%(p<0.05)$. Data is presented as mean \pm standard deviation (SD).

\section{Results}

\section{Patient Cohort}

The characteristics of the present patient cohort are summarized in Table 1. A total of 572 patients were included in this retrospective study. Patients' age ranged from 17 to 87 years (mean $48.2 \pm 12.6$ years). Females were affected more often than male patients. Fifty-four percent of the VS was located on the right side and $46 \%$ on the left side. Tumor sizes were
Table 1 Demographic and tumor characteristics of the patient cohort

\begin{tabular}{ll}
\hline Age (years) & $48.2 \pm 12.6$ \\
& {$[16.8-87.3]$} \\
\hline Gender & $310 / 572(54 \%)$ \\
Female & $262 / 572(46 \%)$ \\
Male & \\
Tumor side & $262 / 572(46 \%)$ \\
Left & $310 / 572(54 \%)$ \\
Right & \\
Tumor size & $37 / 572(7 \%)$ \\
T1 & $123 / 572(22 \%)$ \\
T2 & $239 / 572(42 \%)$ \\
T3 & $173 / 572(30 \%)$ \\
T4 & \\
Positioning & $128(22 \%)$ \\
Supine & $444(78 \%)$ \\
Semi-sitting & \\
STS (min) & $264 \pm 68$ \\
& {$[70-610]$} \\
\hline
\end{tabular}

classified according to the Koos classification. Intracanalicular (T1) VS accounted for 7\% of the tumors, $22 \%$ were T2, $42 \%$ were $\mathrm{T} 3$, and $30 \%$ were $\mathrm{T} 4 \mathrm{VS}$.

\section{Surgical Technique}

While small T1/2 VSs are operated in supine position (SP), we prefer the semi-sitting position (SSP) for large T3/4 tumor. In the present cohort, $78 \%$ surgeries were performed in SSP. Ninety-one percent of T3/4 VSs were operated in SSP. In contrast, only $6 \%$ of T1/2 VS underwent surgery in SSP $\left(X^{2}=354.13, p<0.001\right)$. Mean duration of surgery (skin-to-skin time, STS) was $264 \pm 68$ [70-610] min. Remarkably, there were no significant differences in STS ( $H=3.52 ; p=0.061$; Kruskal-Wallis) comparing surgeries in SP $(248 \pm 47 \mathrm{~min})$ and SSP $(267 \pm 72 \mathrm{~min})$. In contrast, Kruskal-Wallis analysis of variance determined a significant effect of tumor size on STS $(H=80.07 ; p<0.001)$. Dunn's post hoc test confirmed that T4 tumors necessitate significant longer STS than smaller tumors.

\section{Clinical Outcome of Facial Nerve Function}

Most patients (96\%) had no signs of facial weakness on admission. Only 4\% patients suffered from a preoperative facial palsy. Immediately after surgery, more than half of the patients deteriorated one or more grades according to

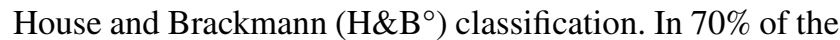
patients, facial function normalized within 3 months. After

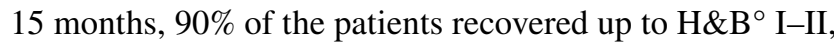
$7 \%$ had $\mathrm{H} \& \mathrm{~B}^{\circ} \mathrm{III}$, and $2 \%$ maintained poor facial function (H\&B ${ }^{\circ}$ IV: Fig. 3). Good results were more evident for small

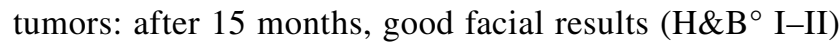


Fig. 3 Outcome of facial nerve function. Pie charts (\% of patients) depict the facial function directly after surgery (postOP), after 3 (3Mo-FU) and 15 months (15Mo-FU). Outcome of facial nerve functions depends on VS tumor size and improves during neurorehabilitation within the first year after surgery. The rate of favorable outcome (i.e., $H \& B^{\circ} 1-2$ ) is indicated with black lines

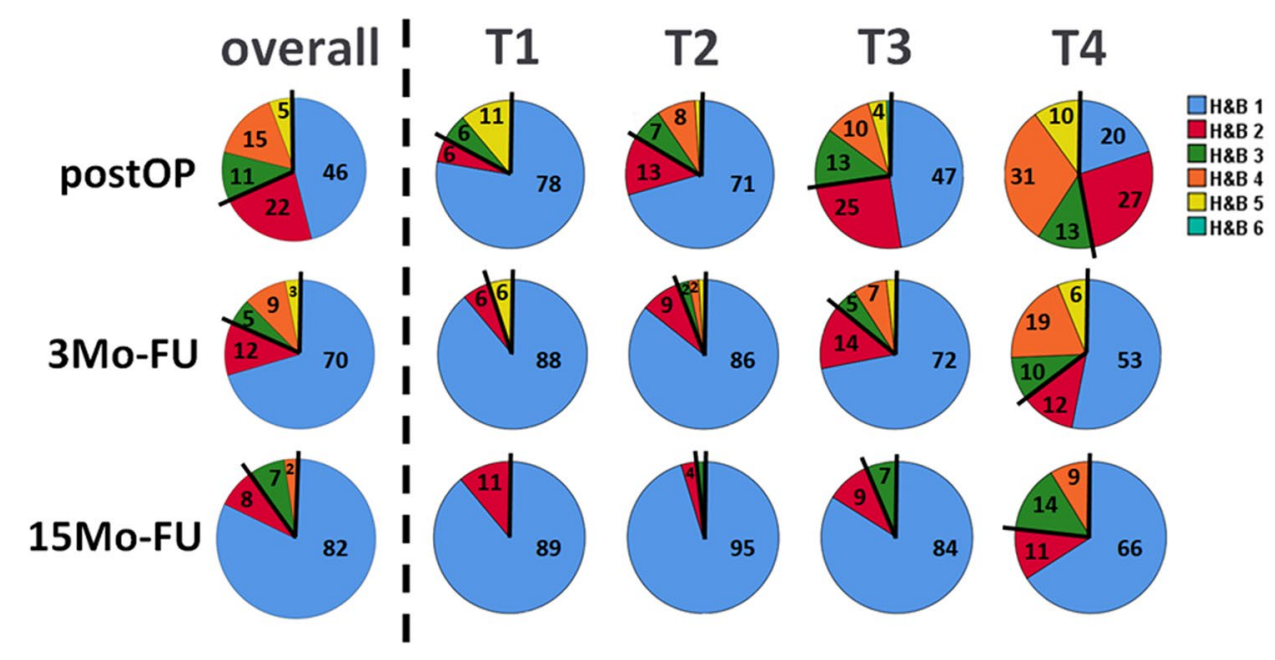

were observed in all patients with T1 VS, in $99 \%$ of the cases with T2 VS, in $93 \%$ of the cases with T3 VS, and in $77 \%$ of the cases with T4 VS (Table 2).

\section{Clinical Outcome of Hearing Function}

Hearing outcome depends on preoperative hearing function and tumor size (Fig. 4). Before surgery, 69\% of the patients had serviceable hearing (i.e., $G \& R^{\circ} 1$ and $G \& R^{\circ} 2$ ). After surgery, overall, $28 \%$ of the patients showed a serviceable hearing, and $48 \%$ of the patients were deaf. Postoperative hearing decreased with increasing tumor size. Small tumor sizes had the best preservation rate (PR) of hearing (85\% for T1) and servable hearing (72\% in T1). In large (T4) cases, PR of servable hearing $\left(\mathrm{G} \& \mathrm{R}^{\circ} 1-2\right)$ dropped to only $15 \%$ (Table 3 ).

\section{Vasoactive Drugs and Hearing}

To support hearing preservation, we use the intraoperative local application of vasoactive drug papaverine (at a dilution of 1:30 in Ringer solution). Here, we report the preliminary results of a first pilot study on the effect of local application of papaverine on hearing preservation. One hundred and three consecutive patients were included. The exclusion criteria were recurrent tumors and NF2-associated VS. In the treatment group, papaverine was used as local intermittent irrigation throughout the entire intradural work on the tumor. The control group received surgery by the same surgeons without the application of papaverine. Out of the 103 cases, $59 \%$ patients had preoperative hearing, and $41 \%$ patients were functionally deaf. In patients with preoperative hearing, cochlear nerve was anatomically preserved in $90 \%$. Thus, 55 patients were eligible for statistical analysis (i.e., patients with preoperative hearing and anatomically preserved hearing nerve after surgery). There were no significant differences in basic demographic (i.e., age, gender, etc.) and tumor (i.e., size) characteristic between the papaverine and control group. Overall, hearing preservation rate in the control group was 52\%, whereas in the papaverine group it was $70 \%\left(X^{2}=0.31, p=0.580\right.$; chi square with Yates correction). However, within patients with postoperatively persevered hearing, the rate of functional hearing $\left(G \& R^{\circ} \mathrm{I}-\mathrm{II}\right)$ was significantly higher in the papaverine $(81 \%)$ than in the control $(30 \%)$ group $\left(X^{2}=4.84, p=0.028\right.$; chi square with Yates correction). In conclusion, there is evidence that papaverine instillation during tumor dissection and cochlear nerve manipulation supports hearing preservation in VS surgery.
Fig. 4 Hearing outcome. Pie charts ( $\%$ of patients) depict the hearing function (according to the G\&R scale) before (preOP) and after surgery (postOP). Hearing outcome depends on preoperative hearing function and VS tumor size. The rate of servable hearing outcome (i.e., $\left.\mathrm{G} \& \mathrm{R}^{\circ} 1-2\right)$ is indicated with black lines

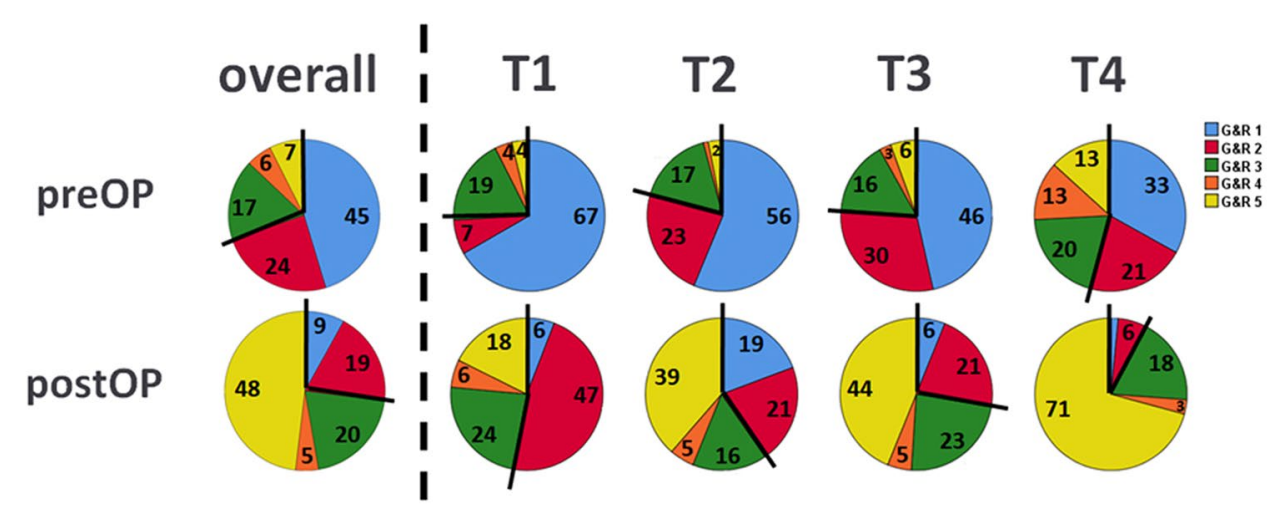


Table 2 Facial outcome. Table shows the percentage (\%) of patients with favorable facial function $\left(\mathrm{H} \& \mathrm{~B}^{\circ} 1-2\right)$ directly after (postOP) surgery as well as after 3 months (3Mo-FU) and 15 months (15Mo-FU) for the whole patient cohort (overall) and separated by tumor size
(T1-T4). Data shows a significant worsening of facial outcome relating to larger tumors. Additionally, it depicts the facial rehabilitation after VS surgery

\begin{tabular}{lllllll}
\hline & Overall & T1 & T2 & T3 & T4 & \\
\hline postOP & $68 \%$ & $84 \%$ & $84 \%$ & $72 \%$ & $47 \%$ & $X^{2}=55.29 ; p<0.001$ \\
3Mo-FU & $82 \%$ & $94 \%$ & $95 \%$ & $86 \%$ & $65 \%$ & $X^{2}=54.35 ; p<0.001$ \\
15 M-FU & $88 \%$ & $100 \%$ & $99 \%$ & $93 \%$ & $76 \%$ & $X^{2}=62.70 ; p<0.001$ \\
& $X^{2}=72.99 ;$ & $X^{2}=5.09 ;$ & $X^{2}=21.87 ;$ & $X^{2}=39.96 ;$ & $X^{2}=25.88 ;$ & $p<0.001$ \\
& $p<0.001$ & $p=0.078$ & $p<0.001$ & $p<0.001$ & $p<1$ \\
\hline
\end{tabular}

\section{Complications}

Surgical mortality was 0 . Complications were detected in $14.5 \%$ of the cases, with most of them being transient. The most common complication was a paradoxical CSF leak. Most of these patients were manageable with a lumbar drainage for 5-7 days. Only a few cases necessitate surgical revision. Symptomatic pneumocephalus due to semi-sitting position occurred in $2.4 \%$ of the cases, treated with subdural puncture of the air. An intradural bleeding was a rare complication in $1.9 \%$ of the cases. Table 4 summarizes the major complications in this series.

\section{Tumor Resection and Recurrence Rates}

Overall, gross-total resection (GTR) was achieved in 93\% of the cases. Complete (GTR) or sub-total (STR) tumor removal was accomplished in all T1-2 tumors. The rates of subtotal tumor removal (STR) or decompressive surgery (DCS) increased with tumor size (Fig. 5A). Major reason for STR was significant decrease of intraoperative facial MEP, in order to avoid anatomical damage to the facial nerve. Overall, the progression-free survival was excellent (Fig. 5B) for the present follow-up period (70 \pm 38 months). Subtotal tumor removal was a strong predictor of recurrence (Table 5). Mean recurrence time was $51 \pm 41$ months.

\section{Discussion}

\section{Surgical Indication}

Generally, the goal of any treatment is cure of a disease while preserving quality of life. Because VSs are slowgrowing tumors, treatment strategy has to be discussed on an individual base. The level of evidence to provide treatment recommendations for VS is relatively low [13••]. The choice of treatment modality will depend basically on clinical presentation, tumor size, patients' age, and expertise of the treating center. Especially in small VS, a watchful MRI follow-up to document tumor growth is recommended before a treatment is initiated. While radiation, particularly radiosurgery, is the treatment of choice in small-growing VS in elderly patients, in younger patients and in patients harboring large tumors, surgery may be the treatment of choice in order to cure or to produce long-term control of the disease. In this regard, our experience does not correspond with the recently published Guidelines of the EANO for VS [13••], since they do not take into consideration patients' characteristics (e.g., age and gender) and basically recommend either observation or radiation - but not surgery - for small VS, and tumor partial resections followed by radiation treatment for large VS. According to the presented data and studies of other high-volume VS centers, microsurgery of small VS provides excellent long-term results and functional
Table 3 Hearing outcome. Table shows the percentage (\%) of patients with any hearing $\left(G \& R^{\circ} 1-4\right)$ and functional hearing $\left(G \& R^{\circ} 1-2\right)$ before (preOP) and after (postOP) surgery for the whole patient cohort (overall) and separated by tumor size (T1-T4). Additionally, hearing preservation rates (PR) of any hearing $\left(G \& R^{\circ} 1-4\right)$ and functional hearing $\left(G \& R^{\circ} 1-2\right)$ of VS surgery are depicted

\begin{tabular}{llllllll}
\hline & & Overall & T1 & T2 & T3 & T4 & \\
\hline preOP & G\&R $^{\circ} \mathbf{1}-\mathbf{4}$ & $93 \%$ & $96 \%$ & $98 \%$ & $94 \%$ & $87 \%$ & $X^{2}=16.03 ; p=0.001$ \\
& $\mathbf{G \& R}^{\circ} \mathbf{1 - 2}$ & $69 \%$ & $74 \%$ & $79 \%$ & $76 \%$ & $54 \%$ & $X^{2}=30.64 ; p<0.001$ \\
postOP & $\mathbf{G \& R}^{\circ} \mathbf{1}-\mathbf{4}$ & $52 \%$ & $82 \%$ & $61 \%$ & $56 \%$ & $29 \%$ & $X^{2}=54.49 ; p<0.001$ \\
& $\mathbf{G \& R}^{\circ} \mathbf{1}-\mathbf{2}$ & $28 \%$ & $53 \%$ & $40 \%$ & $27 \%$ & $8 \%$ & $X^{2}=56.56 ; p<0.001$ \\
PR & $\mathbf{G \& R}^{\circ} \mathbf{1 - 4}$ & $56 \%$ & $85 \%$ & $62 \%$ & $60 \%$ & $33 \%$ & $X^{2}=44.91 ; p<0.001$ \\
& $\mathbf{G \& R}^{\circ} \mathbf{1 - 2}$ & $41 \%$ & $72 \%$ & $51 \%$ & $36 \%$ & $15 \%$ & $X^{2}=42.82 ; p<0.001$ \\
\hline
\end{tabular}



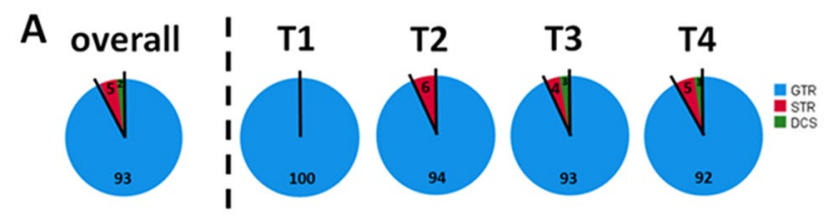

B

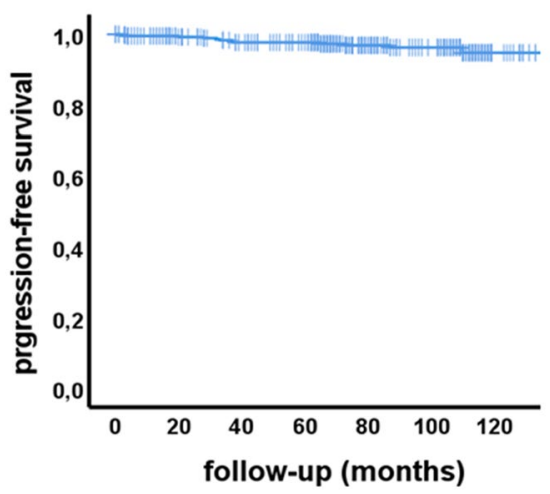

Fig. 5 Tumor resection and recurrence rates. A Pie charts (\% of patients) depict the extent of tumor resection (gross total resection, GTR; subtotal resection, STR; decompressive surgery, DCS) depending on the tumor size. In general, GTR was intended and achieved regularly. B Kaplan-Meyer curve of progression-free survival shows good tumor control for the present follow-up period after VS surgery

outcome comparable to radiosurgery $[14,15]$. Long-term follow-up studies with more than 10 years observation show progression-free survival rate of $92 \%$ following radiosurgery [3]. Facial preservation rate was $97 \%$. This is particularly important for young patients who still have many life decades to live. Moreover, female sex is related to worse progression-free survival following stereotactic radiosurgery at long term [3]. Another critical point on the EANO Guidelines is the overall recommendation for large VS of subtotal or partial tumor removal followed by radiation. According to our experience and others [16••], GTR of large VS is possible in approximately $70-80 \%$ of the cases with good functional results and long-term tumor control. Recurrence of subtotally removed and irradiated VS may become a formidable task for further treatment.

Table 4 Complications

\begin{tabular}{lc}
\hline CSF fistula (treated with lumbar drainage) & $33 / 572(5.8 \%)$ \\
\hline CSF fistula (necessitating surgical revision) & $8 / 572(1.4 \%)$ \\
Tension pneumocephalus & $14 / 572(2.4 \%)$ \\
Intradural bleeding & $11 / 572(1.9 \%)$ \\
SVT & $5 / 572(0.9 \%)$ \\
ARDS & $5 / 572(0.9 \%)$ \\
Epidural bleeding & $2 / 572(0.3 \%)$ \\
Infections & $1 / 572(0.2 \%)$ \\
Lower cranial nerve dysfunction & $1 / 572(0.2 \%)$ \\
\hline
\end{tabular}

Table 5 Recurrence rates

\begin{tabular}{lllllc}
\hline & Overall & GTR & STR & DCS & \\
\hline Recurrence rate & $3.2 \%$ & $1.4 \%$ & $14 \%$ & $75 \%$ & $\begin{array}{c}X^{2}=172.82 ; \\
p<0.001\end{array}$ \\
$\begin{array}{c}\text { Mean recurrence } \\
\text { time (months) }\end{array}$ & $51 \pm 41$ & $71 \pm 53$ & $68 \pm 41$ & $30 \pm 26$ & $\begin{array}{c}H=2.77 ; \\
p=0.322\end{array}$ \\
\hline
\end{tabular}

Latter patients may suffer from fast-growing lesions, facial palsy, and even malignization [3]. Therefore, all efforts should be made to remove these tumors completely at the initial surgery, while maintaining patient's quality of life.

General management options for patients harboring a VS are wait-and-scan, radiotherapy, and microsurgery. Indications for the microsurgical treatment of a patient's VS are (1) large tumor with brainstem compression; (2) growing tumor in a young patient; (3) cystic VS (>50\% of the tumor volume); (4) severe vestibular dysfunction; (5) neurofibromatosis 2 with early partial/subtotal removal; and (6) patient's individual decision.

\section{Surgical Technique}

Opening of the IAC is always the first step of the intradural procedure, unless the VS is giant and blocks the access to the IAC. The reasons for an early opening of the IAC are as follows: (1) the neurovascular structures in the CPA are still protected by the tumor against bone dust, heat, and irrigation; (2) we gain control of the distal part of the facial and the cochlear nerves; (3) in the final stage of the operation, we can follow the facial nerve at the inferior part of the tumor surface from both sides of the meatus, which facilitates facial preservation while removing the last tumor part. The position of the cranial nerves VII and VIII inside the inner auditory canal (IAC) is constant. The transverse crest and the vertical crest (Bill's bar) divide the fundus of the IAC into four parts with the facial nerve in the anterosuperior, the cochlear nerve in the anteroinferior, the superior vestibular in the posterosuperior, and the inferior vestibular nerve in the posteroinferior compartment, respectively. Within the intracisternal part, the tumor displaces facial and cochlear nerve accordingly. The most common location of the facial nerve is along the anterior middle third of the VS and of the cochlear nerve along the anterior inferior part of the VS [17]. However, there is a high variability regarding the course of the facial nerve with approximately $0.6 \%$ of facial nerves facing the posterior aspect of the VS [12]. VS may change the size and shape of the IAC and thus the topography of the surrounding inner ear structures [18]. Particularly, the posterior semicircular canal, the common crus, and the vestibular aqueduct may be under risk when opening the IAC. Their injury significantly correlates with higher risk of postoperative hearing loss [19]. 


\section{Facial Nerve}

Reduced facial nerve function is a major concern of all patients. Nowadays, the chance of facial nerve preservation can be considered very good. Preoperative facial function, tumor size, tumor extension, prior treatment (surgery or radiosurgery), and cystic morphology of the VS are considered predictive factors for postoperative facial nerve function [19]. Considering the high impact of facial nerve dysfunction on the patient's quality of life, it seems reasonable to privilege facial nerve integrity to radical VS resection. The radiosurgical option in case of tumor progress makes this decision reasonable. However, tumor recurrence or regrowth is much higher following STR than GTR or NTR. Nakatomi et al. have shown higher recurrence-free survival following GTR in comparison with STR after 5 years (96\% vs. 47\%), 10 years ( $82 \%$ vs. $17 \%$ ), 15 years ( $73 \%$ vs. $8 \%)$, and 20 years (56\% vs. $0 \%)$ [20].

\section{Hearing}

Samii reports an overall hearing preservation rate of $51 \%$ via the retrosigmoid approach [14]. Long-term serviceable hearing preservation 5 years after retrosigmoid tumor surgery ranges between $83 \%$ [21] and $87 \%$ [22, 23]. However, indicating treatment (microsurgery or radiotherapy) for small VS, one has to consider whether the result of therapy is really superior to the expected natural history. Observing the natural history, patients with small tumors with $100 \%$ speech discrimination (WRS classification) at diagnosis have a chance of $75 \%$ to maintain the good hearing function for 10 years or longer with a waitand-scan strategy [24]. A minor reduction, however, worsens the prognosis: $54 \%$ of the patients with AAO class A hearing lost class A hearing after 10 years [24]. Sughrue et al. evaluated the natural history of VS in an analysis of 982 patients (34 articles) with a follow-up between 26 and 52 months [25]. They found an overall hearing preservation rate of $54 \%$. A statistically significant factor for hearing preservation is an average tumor growth rate $\leq 2.5 \mathrm{~mm} /$ year [25].

\section{Tinnitus}

In our patient cohort, $61.8 \%$ of patients complain about a preoperative tinnitus [26]. A total of $67.5 \%$ of patients with preoperative tinnitus were still suffering after the surgery, while in $32.5 \%$, tinnitus disappeared postoperatively. In contrast, $20.7 \%$ of patients developed a new-onset tinnitus [27]. Notably, patients with sustained tinnitus after VS surgery demonstrate volumetric gray matter changes in various cortical and subcortical brain areas [28]. Regarding the surgical treatment of both preexisting or new-onset tinnitus, Kohno et al. recommend resecting the cochlear nerve in case of preoperative non-functional hearing or BAEP loss during surgery [29].
However, BAEP recordings are not always reliable regarding the real postoperative hearing function [30,31], and cutting the nerve withdraws the option of a cochlear implant, which potentially restores hearing and reduces tinnitus [32, 33].

\section{Postoperative Care}

Many patients operated in the semi-sitting position will present some amount of intracranial air, which is usually not clinically significant. Clinically relevant tension pneumocephalus that requires release via a small burr hole occurs approximately in $3 \%$ of the cases [34].

\section{Conclusion}

In conclusion, surgery of VS has been refined and represents a powerful tool for treatment of the disease. In our opinion surgery is mostly indicated in large VS but also in small VS in young patients and in female patients in whom pregnancy is expected.

Funding Open Access funding enabled and organized by Projekt DEAL.

\section{Declarations}

Conflict of Interest The authors declare that they have no conflict of interest.

Human and Animal Rights and Informed Consent This retrospective study involving human participants was in accordance with the ethical standards of the institutional and national research committee and with the 1964 Helsinki Declaration. The study was approved by the local ethics committee of the Eberhardt Karls University Tuebingen, Germany and fulfills the requirements for retrospective studies of data collected as part of routine diagnosis and treatment.

Open Access This article is licensed under a Creative Commons Attribution 4.0 International License, which permits use, sharing, adaptation, distribution and reproduction in any medium or format, as long as you give appropriate credit to the original author(s) and the source, provide a link to the Creative Commons licence, and indicate if changes were made. The images or other third party material in this article are included in the article's Creative Commons licence, unless indicated otherwise in a credit line to the material. If material is not included in the article's Creative Commons licence and your intended use is not permitted by statutory regulation or exceeds the permitted use, you will need to obtain permission directly from the copyright holder. To view a copy of this licence, visit http://creativecommons.org/licenses/by/4.0/.

\section{References}

Papers of particular interest, published recently, have been highlighted as: 
- Of importance

$\bullet$ Of major importance

1. Ostrom QT, Gittleman H, Liao P, Rouse C, Chen Y, Dowling J, et al. CBTRUS statistical report: Primary brain and central nervous system tumors diagnosed in the United States in 20072011. Neuro Oncol Oxford University Press. 2014;16:1-63.

2. Zanoletti E, Mazzoni A, Martini A, Abbritti RV, Albertini R, Alexandre E, et al. Surgery of the lateral skull base: a 50-year endeavour. Acta Otorhinolaryngol Ital. 2019;39:1-146.

3. Hasegawa T, Kida Y, Kato T, Iizuka H, Kuramitsu S, Yamamoto T. Long-term safety and efficacy of stereotactic radiosurgery for vestibular schwannomas: evaluation of 440 patients more than 10 years after treatment with Gamma Knife surgery. J Neurosurg. 2013;118:557-65.

4.- Watanabe S, Yamamoto M, Kawabe T, Koiso T, Yamamoto T, Matsumura A, et al. Stereotactic radiosurgery for vestibular schwannomas: average 10-year follow-up results focusing on long-term hearing preservation. J Neurosurg. 2016;125:64-72. Findings of this study on radiosurgery of vestibular schwannomas show acceptable tumor control rate, but poor hearing preservation results with long-term follow-up.

5. Ebner FH, Koerbel A, Roser F, Hirt B, Tatagiba M. Microsurgical and endoscopic anatomy of the retrosigmoid intradural suprameatal approach to lesions extending from the posterior fossa to the central skull base. Skull Base. 2009;19:319-23.

6. Scheller C, Wienke A, Tatagiba M, Gharabaghi A, Ramina KF, Ganslandt O, et al. Prophylactic nimodipine treatment and improvement in hearing outcome after vestibular schwannoma surgery: a combined analysis of a randomized, multicenter, Phase III trial and its pilot study. J Neurosurg. 2017;127:137683. Findings of this prospective study show that intravenous nimodipine treatment may improve the chance of hearing preservation in vestibular schwannoma surgery.

7. Erickson NJ, Schmalz PGR, Agee BS, Fort M, Walters BC, McGrew BM, et al. Koos Classification of vestibular schwannomas: a reliability study. Neurosurgery. 2019;85:409-14.

8. Gardner G, Robertson JH. Hearing preservation in unilateral acoustic neuroma surgery. Ann Otol Rhinol Laryngol. 1988;97:55-66.

9. House JW, Brackmann DE. Facial nerve grading system. Otolaryngol Head Neck Surg. Otolaryngol Head Neck Surg 1985, 93:146-147.

10. Campero A, Martins C, Rhoton A, Tatagiba M. Dural landmark to locate the internal auditory canal in large and giant vestibular schwannomas: The Tübingen line. Neurosurgery Neurosurgery. 2011;69:99-102.

11. Shao KN, Tatagiba M, Samii M. Surgical management of high jugular bulb in acoustic neurinoma via retrosigmoid approach. Neurosurgery. 1993;32:32-7.

12. Bae CW, Cho YH, Hong SH, Kim JH, Lee J, Kim CJ. The anatomical location and course of the facial nerve in vestibular schwannomas : a study of 163 surgically treated cases. J Korean Neurosurg Soc. Korean Neurosurgical Society 2007, 42:450-454.

13.••Goldbrunner R, Weller M, Regis J, Lund-Johansen M, Stavrinou P, Reuss D, et al. Eano guideline on the diagnosis and treatment of vestibular schwannoma. Neuro Oncol. 2020;22:31-45. This is a review paper that highlights current strategy in vestibular schwannoma diagnosis and treatment with focus on radiation treatement.

14. Samii M, Gerganov V, Samii A. Improved preservation of hearing and facial nerve function in vestibular schwannoma surgery via the retrosigmoid approach in a series of 200 patients. J Neurosurg. 2006;105:527-35.

15. Samii M, Metwali H, Gerganov V. Efficacy of microsurgical tumor removal for treatment of patients with intracanalicular vestibular schwannoma presenting with disabling vestibular symptoms. J Neurosurg. 2017;126:1514-9.

16.• Starnoni D, Giammattei L, Cossu G, Link MJ, Roche PH, Chacko AG, et al. Surgical management for large vestibular schwannomas: a systematic review, meta-analysis, and consensus statement on behalf of the EANS skull base section. Acta Neurochir. 2020;162(11):2595-617. This consensus paper on behalf of the Skull Base Section of the EANS summarizes the current surgical management of large vestibular schwannomas. The main goal of management of large VS should be improving/maintaining patient's quality of life including facial (and cochlear) function and ensuring best oncological control.

17. Sampath P, Rini D, Long DM. Microanatomical variations in the cerebellopontine angle associated with vestibular schwannomas (acoustic neuromas): a retrospective study of 1006 consecutive cases. J Neurosurg. 2000;92:70-8.

18. Ebner FH, Kleiter M, Danz S, Ernemann U, Hirt B, Löwenheim $\mathrm{H}$, et al. Topographic changes in petrous bone anatomy in the presence of a vestibular schwannoma and implications for the retrosigmoid transmeatal approach. Neurosurgery. 2014;10:481-6.

19. Tatagiba M, Samii M, Matthies C, El Azm M, Schonmayr R. The significance for postoperative hearing of preserving the labyrinth in acoustic neurinoma surgery. J Neurosurg J Neurosurg. 1992;77:677-84.

20. Nakatomi H, Jacob JT, Carlson ML, Tanaka S, Tanaka M, Saito N, et al. Long-term risk of recurrence and regrowth after gross-total and subtotal resection of sporadic vestibular schwannoma. J Neurosurg. 2020;133:1052-8.

21. Nakamizo A, Mori M, Inoue D, Amano T, Mizoguchi M, Yoshimoto $\mathrm{K}$, et al. Long-term hearing outcome after retrosigmoid removal of vestibular schwannoma. Neurol Med Chir (Tokyo). 2013;53:688-94.

22. Mazzoni A, Zanoletti E, Calabrese V. Hearing preservation surgery in acoustic neuroma: long-term results. Acta Otorhinolaryngol Ital. 2012;32:98-102.

23. Ichimasu N, Kohno M, Nakajima N, Matsushima K, Tanaka Y, Tsukahara K, et al. Long-term prognosis of preserved useful hearing after surgery in patients with vestibular schwannoma: a study of 91 cases. Acta Neurochir (Wien). 2020;162:2619-28.

24. Stangerup SE, Thomsen J, Tos M, Cayé-Thomasen P. Long-term hearing preservation in vestibular schwannoma. Otol Neurotol Otol Neurotol. 2010;31:271-5.

25. Sughrue ME, Yang I, Aranda D, Lobo K, Pitts LH, Cheung SW, et al. The natural history of untreated sporadic vestibular schwannomas: a comprehensive review of hearing outcomes Clinical article. J Neurosurg. 2010;112:163-7.

26. Naros G, Sandritter J, Liebsch M, Ofori A, Rizk AR, Del Moro $\mathrm{G}$, et al. Predictors of preoperative tinnitus in unilateral sporadic vestibular schwannoma. Front Neurol. 2017;8:378.

27. Trakolis L, Ebner FH, Machetanz K, Sandritter J, Tatagiba M, Naros G. Postoperative tinnitus after vestibular schwannoma surgery depends on preoperative tinnitus and both pre- and postoperative hearing function. Front Neurol. 2018;9:136.

28. Trakolis L, Bender B, Ebner FH, Ernemann U, Tatagiba M, Naros G. Cortical and subcortical gray matter changes in patients with chronic tinnitus sustaining after vestibular schwannoma surgery. Sci Rep. 2021;11:8411.

29. Kohno M, Shinogami M, Yoneyama H, Nagata O, Sora S, Sato H. Prognosis of tinnitus after acoustic neuroma surgerysurgical management of postoperative tinnitus. World Neurosurg. 2014;81:357-67.

30. Matthies C, Samii M. Management of vestibular schwannomas (acoustic neuromas): the value of neurophysiology for evaluation and prediction of auditory function in 420 cases. Neurosurgery. 1997;40:919-30. 
31. Chovanec M, Zvěřina E, Profant O, Balogová Z, Kluh J, Syka $\mathrm{J}$, et al. Does attempt at hearing preservation microsurgery of vestibular schwannoma affect postoperative tinnitus? Biomed Res Int. 2015, 2015:783169.

32. Holder JT, O'Connell B, Hedley-Williams A, Wanna G. Cochlear implantation for single-sided deafness and tinnitus suppression. Am J Otolaryngol. 2017;38:226-9.

33. Blasco MA, Redleaf MI. Cochlear implantation in unilateral sudden deafness improves tinnitus and speech comprehension: meta-analysis and systematic review. Otol Neurotol. 2014;35:1426-32.
34. Machetanz K, Leuze F, Mounts K, Trakolis L, Gugel I, Grimm $\mathrm{F}$, et al. Occurrence and management of postoperative pneumocephalus using the semi-sitting position in vestibular schwannoma surgery. Acta Neurochir (Wien). 2020;162:2629-36.

Publisher's Note Springer Nature remains neutral with regard to jurisdictional claims in published maps and institutional affiliations. 